\title{
Graphene oxide-assisted synthesis of bismuth nanosheets for catalytic stripping voltammetric determination of iron in coastal waters
}

\author{
Xueping Hu${ }^{1,3}$ - Dawei Pan ${ }^{1,2} \cdot$ Mingyue Lin ${ }^{1,3} \cdot$ Haitao Han ${ }^{1} \cdot \mathrm{Fei}^{4}{ }^{4}$
}

Received: 19 October 2015 / Accepted: 13 December 2015 / Published online: 29 December 2015

(C) Springer-Verlag Wien 2015

\begin{abstract}
The article describes the synthesis of bismuth nanosheets (BiNSs) in the presence of a small quantity of graphene oxide (GO) which is helpful for the formation of twodimensional BiNSs and improves dispersity. The material, when placed on a glassy carbon electrode (GCE), is shown to enable catalytic stripping voltammetric determination of total dissolved iron without the need for adding a complexing agent. The average thickness and length of the BiNSs are 3 to $4 \mathrm{~nm}$ and 100 to $200 \mathrm{~nm}$, respectively. The unique nanostructure of the BiNSs, the ability of Bi to form alloys with metal, and the current amplification of the catalytic system make the modified GCE an excellent choice for electrochemical determination of $\mathrm{Fe}(\mathrm{III})$. Under the optimal conditions, the electrode has a linear response to $\mathrm{Fe}(\mathrm{III})$ in the 0.01 to $20 \mu \mathrm{M}$ concentrations range, with a lower detection limit of $2.3 \mathrm{nM}$.
\end{abstract}

Electronic supplementary material The online version of this article (doi:10.1007/s00604-015-1733-z) contains supplementary material, which is available to authorized users.

Dawei Pan

dwpan@yic.ac.cn

1 Key Laboratory of Coastal Environmental Processes and Ecological Remediation, Yantai Institute of Coastal Zone Research (YIC), Chinese Academy of Sciences (CAS), Shandong Provincial Key Laboratory of Coastal Environmental Processes, Yantai, Shandong 264003, People's Republic of China

2 Department of Earth, Ocean and Ecological Sciences, School of Environmental Science, University of Liverpool, Liverpool L69 3GP, UK

3 University of Chinese Academy of Sciences, Beijing 100049, People's Republic of China

4 College of Chemistry, Chemical Engineering and Materials Science, Shandong Normal University, Jinan, Shandong 250014, People's Republic of China
The electrode was successfully applied to the sensitive determination of $\mathrm{Fe}(\mathrm{III})$ in coastal waters.

Keywords Differential pulse voltammetry · Catalytic current amplification $\cdot$ Scanning electron microscopy $\cdot$ Energy dispersive $\mathrm{X}$-ray spectroscopy

\section{Introduction}

Recently, the interest of the scientific community is aimed at the design and development of electrochemical methods for selective detection of environmentally and biologically relevant metal ions [1]. As one of such metal ions, iron plays a critical role for all living organisms by participating in a wide diversity of metabolic processes, including hemoglobin synthesis, oxygen transport and electron transport [2,3]. Besides, iron is responsible for chlorophyll synthesis, nitrate reduction, and detoxification of reactive oxygen species [4]. The iron in natural water has been regarded as a growth-limiting factor for microorganisms and determines what phytoplankton species dominate $[5,6]$. So, the quantitative determination of total dissolved iron is important for biology as well as the environment. Many techniques have been developed to detect and quantify iron such as atomic absorption spectrometry [7], inductively coupled plasma-mass spectrometry (ICP-MS) [8], inductively coupled optical emission spectrometry [9], flow injection analysis [10] and fluorescence [11]. However, most analytical methods are based on relatively expensive instrument. Moreover, the size and weight of the instruments also restrict their use for in situ determination.

The electrochemical stripping analysis $[12,13]$ is recognized as an extremely sensitive electrochemical technique for iron determination [14]. For many years, the electroanalytical techniques were linked with the use of mercury electrodes as 
working electrode for the determination of iron $[15,16]$, various chelating agents including 2,3-dihydroxynaphthalene (DHN) [17], 1-nitroso-2-naphthol (NN) [18], and 2-(2-thiazolylazo)$p$-cresol (TAC) [16] have been used to accumulate iron complexes on the electrode surface. Considering the drawbacks of mercury electrode, such as toxicity, difficulties in the handling and storage [19], chemically modified electrodes have been developed for iron determination. Among the various modified electrodes, the bismuth-based electrodes seem to be the most promising alternatives owing to their lower mercury toxicity, ability to form alloys with metal and considerably sensitive voltammetric detection. To date, a variety of bismuth-based electrodes, such as bismuth film (BiF) electrodes [20], bismuth bulk film electrode [21], polymer modified bismuth electrode [22], Bi nanotube modified electrode [23] and Bi nanoparticle modified electrode [24] have been developed for analysis of metal ions and organic compounds, but most ubiquitous and widely used configuration in stripping analysis is the bismuth film electrode. However, the utilization of bismuth-based electrode for the determination of $\mathrm{Fe}$ (III) has attracted only limited attention. Ex-situ deposited bismuth film electrodes [25, 26] and tin-bismuth alloy electrode [27] have been developed for iron determination with the aid of chelating agents. Considering the fascinating properties of nanomaterial, bismuth with a nanostructure may be a good candidate to modify the electrode with the better analytical selectivity and sensitivity in the determination of $\mathrm{Fe}$ (III) because of its various shapes, sizes, large surface areas and excellent electronic properties.

In this study, a bismuth nanosheet (BiNS) material was successfully synthesized with the precursor of the carbon nanomaterial graphene oxide (GO). It is a good notion to use the $\mathrm{GO}$ as a precursor to synthesize a nano-structural material. The thickness of the nanosheet is about $3 \sim 4 \mathrm{~nm}$, which resulting in a huge surface areas and more active sites of this bismuth material. The BiNS modified glassy carbon electrode (BiNS/GCE) has been used for the determination of Fe(III) without using any complex agents. A number of factors had been optimized to obtain the best analytical for $\mathrm{Fe}(\mathrm{III})$ and the linear response was in the concentration range of $0.01-20 \mu \mathrm{M}$ with a detection limit of $2.3 \mathrm{nM}$. Additionally, the BiNS/GCE was also successfully applied to the sensitive determination of $\mathrm{Fe}(\mathrm{III})$ in real coastal waters with satisfactory results.

\section{Experimental}

\section{Reagents}

All chemicals and reagents were of analytical grade. GO was supplied by Nanjing Jcnano Technology Company (Nanjing, China. http://www.jcnano.com/). Bismuth (III) nitrate pentahydrate was purchased from Sinopharm Chemical Reagent (Shanghai, China. http://www.sinoreagent.com.cn/).
The percentage of hydrazine hydrate was $80 \%$. Iron standard solution was purchased from Acros Organics (USA. http://www.acros.com/). All other chemicals were of analytical reagents and used without further purification. All experiments were conducted at room temperature $\left(25^{\circ} \mathrm{C}\right)$ and all solutions were prepared using deionized water $(\rho=18$. $2 \mathrm{M} \Omega \mathrm{cm}$ ) from Pall Cascada laboratory water system (USA. http://www.pall.com/main/home.page).

\section{Apparatus}

The morphology and elemental composition of the BiNSs were characterized by scanning electron microscopy (SEM Hitachi S-4800 microscope, Japan. http://www.hitachi.com/) and energy dispersive X-ray spectroscopy (EDX HORIBA EX-350 Japan. http://www.horiba.com/), respectively. Inductively coupled plasma-mass spectrometry (ICP-MS, ELAN DRC, Perkin Elmer Instruments, USA. http://www. perkinelmer.com/) was used for comparative testing. $\mathrm{pH}$ measurements were performed using an E-201-C Model pH meter (Shanghai Leici Instrument Factory, China. http://www. lei-ci.com/). Electrochemical Work Station (CHI 660D, Chenhua Instruments, Shanghai, China. http://chi.instrument. com.cn/) was used throughout in all electrochemical experiments. A conventional three electrode system consisting of a modified glassy carbon working electrode (GCE, $3 \mathrm{~mm}$ in diameter), a platinum foil auxiliary electrode, and a silver chloride reference electrode $(\mathrm{Ag} /$ $\mathrm{AgCl}, 3 \mathrm{M}$ ) was employed. All potentials were measured with respect to the $\mathrm{Ag} / \mathrm{AgCl}$ reference electrode.

\section{Synthesis of bismuth nanosheets}

Firstly, stock bismuth nitrate solution was prepared by dissolving bismuth nitrate with distilled water, while GO was dispersed in distilled water. Secondly, stock bismuth nitrate solution and GO solution were mixed under stirring. The final mixture solution contains $0.01 \mathrm{M}$ bismuth nitrate and GO $(1.5 \% \mathrm{w} / \mathrm{w})$. Then $20 \mathrm{~mL} 0.02 \mathrm{M}$ hydrazine hydrate was added into the $50 \mathrm{~mL}$ mixed solution, the procedure was accompanied by the formation of insoluble substances. The $\mathrm{pH}$ of the above solution was adjusted to $12 \sim 12.5$ with ammonium hydroxide immediately with vigorous stirring for $30 \mathrm{~min}$. Later, the mixed solution would be transferred into the Teflon reaction kettle and kept in a thermostat at $120{ }^{\circ} \mathrm{C}$ for $12 \mathrm{~h}$. Finally, the product was filtered with $0.45 \mu \mathrm{m}$ membrane filters and washed several times with $1 \mathrm{M} \mathrm{HCl}$, distilled water and absolute ethanol exhaustively. After the precipitate had been dried under vacuum at $60{ }^{\circ} \mathrm{C}$ for $4 \mathrm{~h}$, the BiNSs were finally obtained. By contrast, Bi material was synthesized in the same way just with the absence of GO and the reduced graphene oxide (RGO) was synthesized with the absence of bismuth nitrate. 


\section{Preparation of the modified electrode}

Prior to use, bare GCE was polished on aqueous alumina slurry $(0.3$ and $0.05 \mu \mathrm{m})$, and washed with deionized water thoroughly, then ultrasonicated for $1 \mathrm{~min}$. After that, the GCE was subjected to potential cycling $\left(-0.2 \sim 0.8 \mathrm{~V}, 10 \mathrm{mV} \mathrm{s}^{-1}\right)$ in $0.05 \mathrm{M} \mathrm{HCl}$ until reproducible cyclic voltammograms were obtained. Subsequently, the slurry of BiNSs was prepared by mixing $2 \mathrm{mg}$ BiNSs and $1 \mathrm{~mL}$ mixture solution of $30 \%$ ethanol $+63.75 \% \mathrm{H}_{2} \mathrm{O}+6.25 \%$ Nafion under sonicating for $30 \mathrm{~min}$ [23]. Finally, $5 \mu \mathrm{L}$ slurry was added onto the surface of GCE and dried using an infrared lamp to get the BiNS/ GCE. Bi material modified electrode $(\mathrm{Bi} / \mathrm{GCE})$ and reduced graphene oxide modified electrode (RGO/GCE) were prepared at the same way.

\section{Electrochemical analysis procedure}

Unless otherwise stated, $0.05 \mathrm{M} \mathrm{HCl}$ was used as the supporting electrolyte for Fe(III) determination. Before the determination, the modified electrode will be immersed in the electrolyte containing $\mathrm{Fe}(\mathrm{III})$ and $\mathrm{KBrO}_{3}$ for a while with stirring to let the $\mathrm{Fe}(\mathrm{III})$ absorbed onto the surface of the electrode. Then, the reduction responses of Fe(III) to Fe (II) at the electrodes were investigated by differential pulse voltammetry (DPV), using the following parameters: initial potential of $0.8 \mathrm{~V}$, final potential of $0.2 \mathrm{~V}$, amplitude of $0.05 \mathrm{~V}$, potential incremental of $0.004 \mathrm{~V}$, pulse width of $0.2 \mathrm{~s}$, pulse period of $0.5 \mathrm{~s}$ and quiet time of $2 \mathrm{~s}$.

\section{Preparation of real samples}

Coastal river water samples were collected from two local rivers that flow into the Bohai Sea (Shandong province, China). Coastal seawater samples were collected from the Huanghai Sea (Shandong province, China). All the water samples were stored in polyethylene bottles after filtration and kept at $4{ }^{\circ} \mathrm{C}$ until determination. A certain amount of $\mathrm{HNO}_{3}$ and $\mathrm{H}_{2} \mathrm{O}_{2}$ was added to adjust the $\mathrm{pH}$ of water samples less than 2.0, then digested samples in quartz tubes using a $500 \mathrm{~W}$ UV lamp (Metrohm MVA-UV 705, Switzerland. http://www.metrohm. $\mathrm{com} /$ ). Voltammetric measurements were performed on water samples diluted with the $0.05 \mathrm{M} \mathrm{HCl}$ supporting electrolyte.

\section{Results and discussion}

\section{Choice of materials}

Various materials such as Nafion [28], gold nanoparticles [29], multi-wall carbon nanotubes [30], methylene blue [31], bismuth [25] and titanium carbide nanoparticles [32] have been used to increase the selectivity and sensitivity of Fe(III) determination. Among them, bismuth-based electrodes have gained widely acceptance for trace determination of iron instead of the mercury electrode, owing to its low toxicity, the ability to form alloys with many heavy metals, simple preparation, wide potential window, and insensitivity to dissolved oxygen. More importantly, bismuth has shown good electrochemical signals to $\mathrm{Fe}(\mathrm{III})$ in previous reports $[25,26]$.

Nanomaterials have been widely used for construction of electrochemical sensors because of their various shapes, sizes, and compositions which can increase the analytical selectivity and sensitivity. In particular, GO has been a hot research topic in the past few years for its unique construction. Besides, large number of functional groups in the surface of GO can be interactive with bismuth ions, which facilitate the formation of BiNSs. BiNSs material that synthesized with the precursor of GO not only possesses the nanostructure that similar with GO but also has a high percentage of bismuth content. Excellent absorption property and large surface areas from the unique structure combined with the abilities of bismuth to form alloys with metal ions make the BiNS a potential material for the determination of $\mathrm{Fe}(\mathrm{III})$.

\section{Characterization of bismuth nanosheets}

The morphologies and elemental composition of the synthesized BiNSs were characterized by SEM and EDS, respectively. Figure 1 shows SEM images at different magnifications (a, b), Bi material synthesized without precursor (c), and the EDS spectrogram of BiNSs (d). From the Fig. 1a and b, it can be seen that the BiNSs based on the GO as the precursor were uniformly distributed with a thickness of $3 \sim 4 \mathrm{~nm}$. More interestingly, the diameter of BiNSs among different batches is consistent to some extent and the average diameter is about $100 \sim 200 \mathrm{~nm}$. Besides, the thinness of BiNSs is almost the same at about $3 \sim 4 \mathrm{~nm}$. This method for the synthesis of BiNSs has a good reproducibility. With the absence of GO, the bismuth material in the Fig. 1c were aggregated with a big size. Compared with Fig. 1a and c, it is easily to find out that the addition of GO makes the material having good dispersity and uniform shape. GO is a two-dimensional carbon-based monolayer with a lot of oxygen-containing functional groups on its surface. It is supposed that when a bit amount of GO was added into the bismuth nitrate solution, the existence of oxygen-containing functional groups makes the GO becoming electronegative and it can adsorb the positively charged $\mathrm{Bi}(\mathrm{III})$ because of the electrostatic interaction. When the GO and $\mathrm{Bi}(\mathrm{III})$ were reduced in the reactor simultaneously, because of the percentage of GO was quite low, it would be reduced to many layered reduced graphene oxide and the surrounding $\mathrm{Bi}(\mathrm{III})$ would be reduced and coalesced forming a similar two-dimensional structure. The signals of $\mathrm{C}$ and $\mathrm{O}$ in Fig. 1d were due to the RGO. Strong Bi signals were detected and the values of the signal can prove that the main 
Fig. 1 SEM micrographs of the BiNSs composite $(\mathbf{a}, \mathbf{b}), \mathrm{Bi}$ composite (c) and EDX spectrum of BiNSs composite (d)
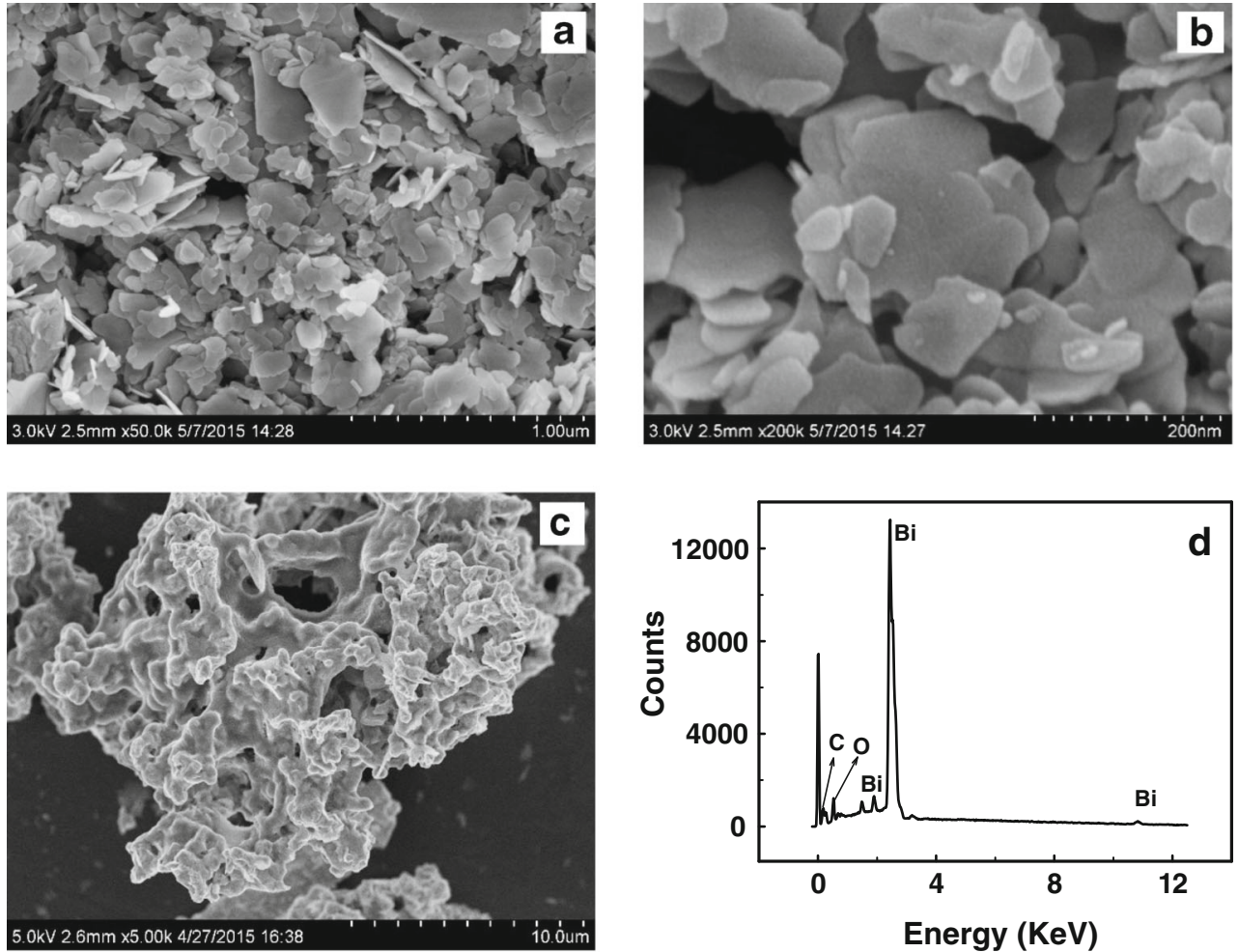

components of the BiNSs were bismuth with the weight percentage of $87.96 \%$.

\section{Electrochemical responses of $\mathrm{Fe}(\mathrm{III})$ on bismuth nanosheets modified glassy carbon electrode}

A comparison of the DPV current densities of Fe(III) at different modified GCE has been investigated. From the Fig. 2, no reduction peak of $\mathrm{Fe}(\mathrm{III})$ was obtained at bare GCE, while the obvious reduction peak of $\mathrm{Fe}$ (III) at almost $0.58 \mathrm{~V}$ (vs. $\mathrm{Ag} /$ $\mathrm{AgCl})$ can be obtained at $\mathrm{Bi} / \mathrm{GCE}$ and $\mathrm{BiNS} / \mathrm{GCE}$, indicating the bismuth has a good response to the determination of $\mathrm{Fe}(\mathrm{III})$ [25]. The reduction potential of $\mathrm{Fe}(\mathrm{III})$ at BiNS/GCE

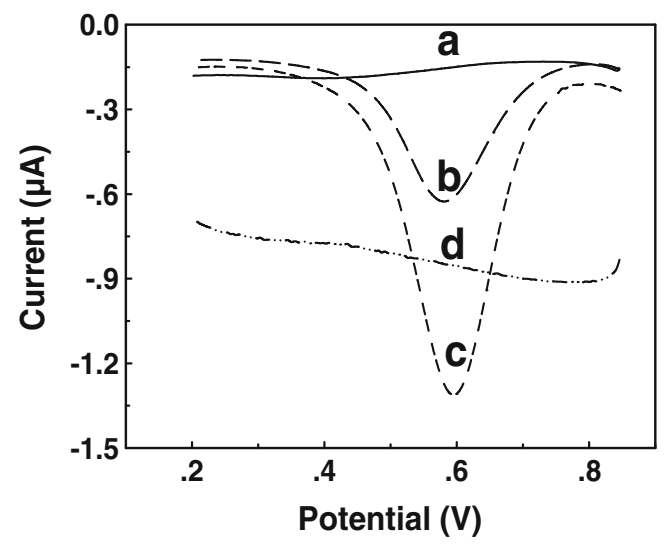

Fig. 2 DPV measurements from 0.8 to $0.2 \mathrm{~V}$ (vs. $\mathrm{Ag} / \mathrm{AgCl}$ ) at bare $\mathrm{GCE}$ (a), $\mathrm{Bi} / \mathrm{GCE}(\mathbf{b}), \mathrm{BiNS} / \mathrm{GCE}$ (c) and $\mathrm{RGO} / \mathrm{GCE}$ (d) in $0.05 \mathrm{M} \mathrm{HCl}$ solution containing $5 \mu \mathrm{M}$ Fe(III) and $\mathrm{Bi} / \mathrm{GCE}$ was consistent with the previous report [26] but more positive than that at other electrodes, which means the $\mathrm{Fe}(\mathrm{III})$ was easier to be reduced at bismuth based electrode due to the excellent properties of bismuth. The different reduction potentials of $\mathrm{Fe}$ (III) may be attributed to various electrode materials. A larger peak current can be obtained at BiNS/ GCE rather than that at $\mathrm{Bi} / \mathrm{GCE}$. The material which was synthesized by using GO as a precursor has uniform nanostructure and large active areas than the aggregated bismuth material. To explore the functions of GO in the synthetic material, a RGO modified GCE was prepared. Large background current densities with almost no peak current of Fe(III) can be seen at RGO modified GCE (Fig. 2d), which further illustrates that RGO has almost no response of Fe(III) [31]. However, during the process of bismuth synthesis, the GO can act as a synthesis precursor which is helpful for the formation of twodimensional BiNSs and the increase of the dispersity. It is bismuth with the uniform nanostructure that plays an important role in the determination of $\mathrm{Fe}(\mathrm{III})$. Besides, the effects of the ratio of GO and bismuth on the response of $\mathrm{Fe}(\mathrm{III})$ were also explored. With the increasing proportion of GO, the peak current of $\mathrm{Fe}(\mathrm{III})$ will decrease, which also indicated that the BiNSs play an important role in the determination of Fe(III) instead of GO.

\section{Mechanism of $\mathrm{Fe}(\mathrm{III})$ determination}

To obtain the reduction mechanism of $\mathrm{Fe}(\mathrm{III})$ at the $\mathrm{BiNS} / \mathrm{GCE}$, the effect of scan rate on the reduction current 


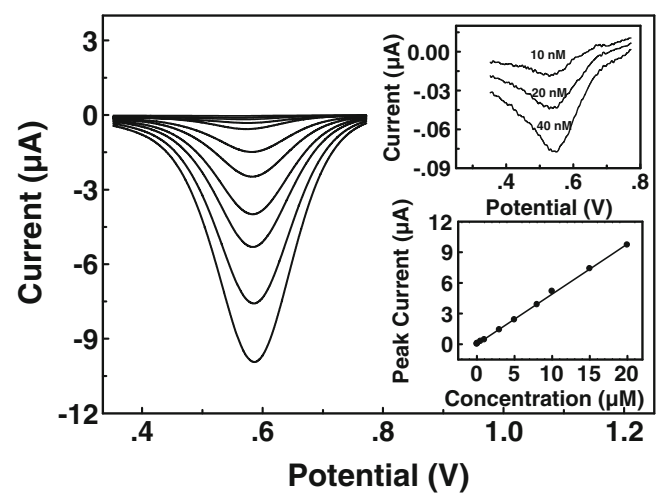

Fig. 3 Voltammetric responses (vs. $\mathrm{Ag} / \mathrm{AgCl}$ ) of $\mathrm{Fe}(\mathrm{III})$ at $\mathrm{BiNS} / \mathrm{GCE}$, where the concentrations of $\mathrm{Fe}(\mathrm{III})$ are from $10 \mathrm{nM}$ to $20 \mu \mathrm{M}$ in $0.05 \mathrm{M}$ $\mathrm{HCl}$ with $6 \mathrm{mM} \mathrm{KBrO}_{3}$. The insets show the voltammetric responses of $10,20,40 \mathrm{nM} \mathrm{Fe}(\mathrm{III})$ (top) and the calibration curve of Fe(III) in the linear range (bottom)

of Fe(III) was studied by linear sweep voltammetry. Cathodic peak current increased gradually and was linear with the increasing scan rate from 25 to $200 \mathrm{mV} \mathrm{s}^{-1}$. The regression equation is expressed as $i_{p}=0.0162 v+0.1349$, indicating that the electro-reduction reactions of iron at the BiNS/ GCE were typical adsorption-controlled process. The occurrence of adsorption was attributed to the adsorptive property of nanomaterial. On the other hand, the presence of $\mathrm{KBrO}_{3}$ in the supporting electrolyte may result in an enhancement of the $\mathrm{Fe}(\mathrm{III})$ response. The reduction and its catalytic regeneration can be described as follows: $\mathrm{Fe}(\mathrm{III})$ will become $\mathrm{Fe}(\mathrm{II})$ through the electrochemical reduction process and then the $\mathrm{Fe}$ (II) can be oxidized to $\mathrm{Fe}(\mathrm{III})$ quickly at the presence of oxidant $[25,33]$. The reoxidized Fe(III) will be reduced on the surface of the modified electrode and contributes again to the reduction current, causing a greatly improved sensitivity.

\section{Optimization of method}

The following parameters were optimized: (a) Type of electrolytes; (b) Sample pH value; (c) Adsorption time; (d) Concentration of $\mathrm{KBrO}_{3}$; Corresponding data and Figures are given in the Electronic Supporting Material. The following experimental conditions were found to give best results: (a) $\mathrm{HCl}$ solution; (b) A sample $\mathrm{pH}$ value of 1.3; (c) Adsorption time of $180 \mathrm{~s}$; (d) Concentration of $\mathrm{KBrO}_{3}$ of $6 \mathrm{mM}$.

\section{Calibration plot}

The calibration curve of the Fe(III) determination was derived from the DPVs obtained at the BiNS/GCE in catalytic system under the optimum conditions. As it shown in Fig. 3, the resulting calibration plots had a favourable linear in the range of $0.01 \sim 20 \mu \mathrm{M}$. The equation for linear regression was $i_{p}=-0.4900 C-0.0069$ with the linear correlation coefficient of 0.999 . The detection limit of Fe(III) at a BiNS modified GCE was $2.3 \mathrm{nM}(\mathrm{s} / \mathrm{n}=3)$. Furthermore, comparisons about iron determination by electrochemical methods and other analytical methods were presented in Table 1. Electrochemical methods have good performances for its inexpensive instrument, simple procedure and relatively lower detection limit. In the electrochemical determination of iron, the absence of complex agents always accompanies with rarely high detection limit. However, our modified electrode exhibits a wide linear range and relatively lower detection limit without the utilization of any complex agents. From the discussed above, the BiNS/GCE may be a good choice for iron determination in coastal water because of its satisfactory results.

Table 1 Comparison of the electrochemical methods with other methods for iron determination

\begin{tabular}{|c|c|c|c|c|c|}
\hline Methods & Electrode & Agents & $\begin{array}{l}\text { Linear range } \\
(\mathrm{nM})\end{array}$ & $\begin{array}{l}\text { LOD } \\
(\mathrm{nM})\end{array}$ & Reference \\
\hline ICP-MS & No & Nitrilotriacetic acid chelating resin & $0.02 \sim 0.07$ & 0.02 & [8] \\
\hline Fluorescence & No & Salicylic acid & $1000 \sim 10,000$ & $50 \sim 357$ & [11] \\
\hline $\mathrm{DLLME}^{\mathrm{a}} / \mathrm{UV}-\mathrm{Vis}$ & No & 5-Br-PADAP ${ }^{d}$ & $90 \sim 7000$ & 27 & [34] \\
\hline Spectrophotometry and flow injection analysis & No & Ascorbic acid DPA-4-SA ${ }^{\mathrm{e}}$ & $90 \sim 357$ & 18 & {$[10]$} \\
\hline DPV & BiF modified GCE & Triethanolamine & $25 \sim 1000$ & 7.7 & {$[25]$} \\
\hline $\mathrm{CSV}^{\mathrm{b}}$ & BiF modified GCE & 1-(2-piridylazo)-2-naphthol & $16 \sim 1070$ & 1.78 & {$[26]$} \\
\hline DPV & $\mathrm{GO} / \mathrm{MB} / \mathrm{AuNPs} / \mathrm{GCE}^{\mathrm{c}}$ & No & $300 \sim 100,000$ & 15 & {$[31]$} \\
\hline DPV & BiNS modified electrode & No & $10 \sim 20,000$ & 2.3 & This work \\
\hline
\end{tabular}

\footnotetext{
${ }^{a}$ Dispersive liquid-liquid microextraction

${ }^{\mathrm{b}}$ Cathodic stripping voltammetry

${ }^{\mathrm{c}}$ Reduced graphene oxide/methylene blue/gold nanoparticles composite modified glassy carbon electrode

d 2-(5-bromo-2-pyridylazo)-5-diethylaminophenol

${ }^{\mathrm{e}}$ Diphenylamine-4-sulfonic acid sodium salt
} 


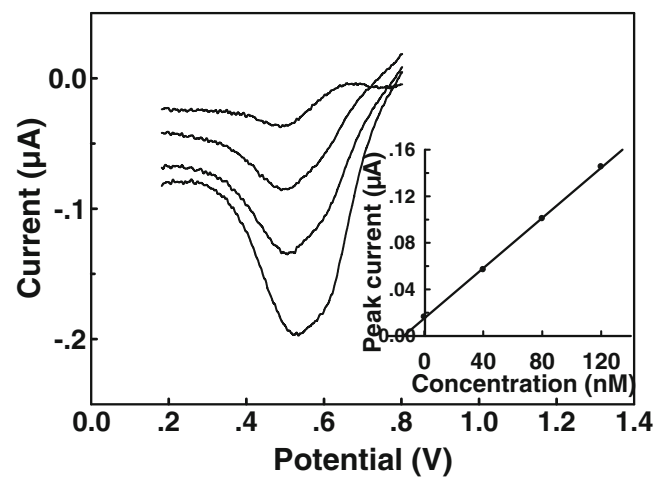

Fig. 4 Typical voltammograms for the determination (vs. $\mathrm{Ag} / \mathrm{AgCl}$ ) of $\mathrm{Fe}(\mathrm{III})$ in coastal river sample 1 by the standard addition method. The concentrations of the addition of Fe(III) are $0,40,80,120 \mathrm{nM}$ (from top to bottom), respectively. Insert: the corresponding linear analytical curve for $\mathrm{Fe}(\mathrm{III})$ determination

\section{Reproducibility, repeatability and selectivity}

During the determination of $\mathrm{Fe}(\mathrm{III})$, parts of the material seem to be oxidized and affect the reduction of Fe(III). However, the reproducibility of the BiNS/GCE was investigated in $1 \mu \mathrm{M}$ $\mathrm{Fe}(\mathrm{III})$ by ten independently modified electrodes prepared with the same method and the relative standard deviation (RSD) of the peak current was $4.2 \%$. The repeatability of the developed method was also evaluated by detecting a certain amount of $\mathrm{Fe}$ (III) in a catalytic system for one electrode in five measurements and the RSD was $3.8 \%$. The modified GCE exhibited good reproducibility and repeatability, which may be attributed to the quick determination procedure and the hardly loss of $\mathrm{Bi}$.

To investigate the effects of possible interfering species for the determination of $\mathrm{Fe}(\mathrm{III})$, various potentially interfering ions were added into the catalytic system. 50 -fold $\mathrm{Mn}^{2+}$ and $\mathrm{Al}^{3+}, 30$-fold $\mathrm{Cu}^{2+}, \mathrm{Pb}^{2+}$ and $\mathrm{Cr}^{3+}, 10$-fold $\mathrm{Zn}^{2+}$ and $\mathrm{Cd}^{2+}$ did not affect the determination of $\mathrm{Fe}$ (III) $(<5 \%$ of response current change). Besides, the effects of organic species were also evaluated, such as humic substances and catechol which are common existed in the natural environment and the result shows that 20-fold humic substances and catechol did not affect the determination of Fe(III). Although many substances can be adsorbed on the electrode, but the reduction potential of
$\mathrm{Fe}(\mathrm{III})$ is constant and not influenced by other substances in certain cases at BiNS/GCE. However, the fact is not denied that other ions may be possibly reduced at this material due to the less selectivity of Bi.

\section{Practical applications}

To evaluate the practical application of this modified electrode, the BiNS/GCE was firstly used for the total dissolved iron determination in coastal water samples. Coastal river water samples were diluted 500 times and analyzed by using the standard addition method under the optimal conditions. The adsorptive cathodic stripping curves of the coastal river water 1 were shown in Fig. 4. Peak currents were linear with the concentrations (the inset in Fig. 4) and the equation was $i p=-0.0012 C-0.0169$ with a correlation coefficient of 0.997 . The concentration of total dissolved iron was calculated to be $16.9 \mathrm{nM}$ and the result was in agreement with the value detected by ICP-MS, indicating the capability of the BiNS/GCE for iron determination. Besides, the concentrations of $\mathrm{Fe}(\mathrm{III})$ in other samples were estimated and the corresponding results were displayed in Table 2. Good quantitative recoveries and precision of the results implied the present method was reliable and suitable for the determination of $\mathrm{Fe}(\mathrm{III})$ in real samples.

\section{Conclusions}

In summary, BiNSs have been synthesized by using GO as the precursor. The BiNS modified GCE has successfully applied to the determination of total dissolved iron in real coastal water samples. However, during the determination of $\mathrm{Fe}(\mathrm{III})$, parts of the material seem to be oxidized at high positive potential. The determination needs to be operated as quickly as possible. Considering the characteristics of BiNSs, such as good adsorptive property, environmental friendliness and ability to form an alloy with metal ions, this Bi-based material has a promising use in accurate electroanalysis of metal ions and might have more potential applications in negative potential.

Table 2 Results of real sample analysis at BiNS/GCE

\begin{tabular}{|c|c|c|c|c|}
\hline Real samples & $\mathrm{Fe}(\mathrm{III})$ added (nM) & Detected by this method (nM) & Detected by ICP-MS ${ }^{\mathrm{a}}(\mathrm{nM})$ & Recovery (\%) \\
\hline Coastal river water 1 & 0 & $16.96 \pm 1.40$ & 17.00 & - \\
\hline Coastal river water 2 & 0 & $23.46 \pm 1.72$ & 23.58 & - \\
\hline \multirow[t]{3}{*}{ Coastal sea water } & 0 & Not detected & - & - \\
\hline & 50 & $48.5 \pm 2.8$ & - & 97.0 \\
\hline & 100 & $97.6 \pm 3.2$ & - & 97.6 \\
\hline
\end{tabular}

${ }^{\text {a }}$ As a comparison, the results were obtained by the actual concentration from ICP-MS determination divided by 500 
Acknowledgments This work was financially supported by the National Natural Science Foundation of China (41276093), the Youth Innovation Promotion Association (2011170) and Outstanding Young Scientists of CAS.

Compliance with ethical standards The authors declare that they have no competing interests.

\section{References}

1. D'Autréaux B, Tucker NP, Dixon R, Spiro S (2005) A non-haem iron centre in the transcription factor $\mathrm{N}$ or $\mathrm{R}$ senses nitric oxide. Nature 437:769-7725

2. Liu X, Theil EC (2005) Ferritins: dynamic management of biological iron and oxygen chemistry. Acc Chem Res 38:167-175

3. Sean R, Lynch MD (2005) The impact of iron fortification on nutritional anaemia. Best Pract Res Clin Haematol 18:333-346

4. Sunda GW, Huntsman SA (1995) Iron uptake and growth limitation in oceanic and coastal phytoplankton. Mar Chem 50:189-206

5. Coale KH, Johnson KS, Fitzwater SE, Gordon RM, Kudela R (1996) A massive phytoplankton bloom induced by an ecosystem-scale iron fertilization experiment in the equatorial Pacific Ocean. Nature 383:495-501

6. Wells ML, Trick CG (2004) Controlling iron availability to phytoplankton in iron-replete coastal waters. Mar Chem 86:1-13

7. Freschi GP, Freschi CD, Neto JG (2008) Evaluation of different rhodium modifiers and coatings on the simultaneous determination of $\mathrm{As}, \mathrm{Bi}, \mathrm{Pb}, \mathrm{Sb}, \mathrm{Se}$ and of $\mathrm{Co}, \mathrm{Cr}, \mathrm{Cu}, \mathrm{Fe}, \mathrm{Mn}$ in milk by electrothermal atomic absorption spectrometry. Microchim Acta 161:129135

8. Jong J, Schoemann V, Lannuzel D, Tison JL, Mattielli N (2008) High-accuracy determination of iron in seawater by isotope dilution multiple collector inductively coupled plasma mass spectrometry (ID-MC-ICP-MS) using nitrilotriacetic acid chelating resin for pre-concentration and matrix separation. Anal Chim Acta 623: 126-139

9. Yang C, Hu Z, Yang J, Gao H (2012) Novel phenyl-iminodiacetic acid grafted multiwalled carbon nanotubes for solid phase extraction of iron, copper and lead ions from aqueous medium. Microchim Acta 176:359-366

10. Asan A, Andac M, Isildak I, Tinkilic N (2008) Flow injection spectrophotometric determination of iron(III) using diphenylamine-4sulfonic acid sodium salt. Chem Pap 62:345-349

11. Cha KW, Park KW (1998) Determination of iron(III) with salicylic acid by the fluorescence quenching method. Talanta 46:1567-1571

12. Lu G, Yao X, Wu X, Zhang T (2001) Determination of the total iron by chitosan-modified glassy carbon electrode. Microchim J 69:81-87

13. Lu M, Compton RG (2013) Voltammetric determination of iron(III) in water. Electroanalysis 25:1123-1129

14. Mashhadizadeh MH, Shosei IS, Monadi N (2004) A novel ion selective membrane potentiometric sensor for direct determination of $\mathrm{Fe}(\mathrm{III})$ in the presence of $\mathrm{Fe}(\mathrm{II})$. Talanta 64:1048-1052

15. Van den Berg CMG (2006) Chemical speciation of iron in seawater by cathodic stripping voltammetry with dihydroxynaphthalene. Anal Chem 78:156-163

16. Croot PL, Johansson M (2000) Determination of iron speciation by cathodic stripping voltammetry in seawater using the competing ligand 2-(2-Thiazolylazo)-p-cresol (TAC). Electroanalysis 12: 565-576

17. Obata H, Van den Berg CMG (2001) Determination of picomolar levels of iron in seawater using catalytic cathodic stripping voltammetry. Anal Chem 73:2522-2528
18. Gledhill M, Van den Berg CMG (1994) Determination of complexation of iron(III) with natural organic complexing ligands in seawater using cathodic stripping voltammetry. Mar Chem 47:41-54

19. Wang J (2005) Stripping analysis at bismuth electrodes: a review. Electroanalysis 17:1341-1346

20. Wang ZQ, Liu G, Zhang LN, Wang H (2012) A bismuth modified hybrid binder carbon paste electrode for electrochemical stripping detection of trace heavy metals in soil. Int J Electrochem Sci 7: 12326-12339

21. Pauliukaite R, Hočevar SB, Ogorevc B, Wang J (2004) Characterization and applications of a bismuth bulk electrode. Electroanalysis 16:719-723

22. Torma F, Kádár M, Tóth K, Tatár E (2008) Nafion/2,2-bipyridylmodified bismuth film electrode for anodic stripping voltammetry. Anal Chim Acta 619:173-182

23. Li Y, Sun G, Zhang Y, Ge C, Bao N, Wang Y (2014) A glassy carbon electrode modified with bismuth nanotubes in a silsesquioxane framework for sensing of trace lead and cadmium by stripping voltammetry. Microchim Acta 181:751-757

24. Lee GJ, Lee HM, Rhee CK (2007) Bismuth nano-powder electrode for trace analysis of heavy metals using anodic stripping voltammetry. Electrochem Commun 9:2514-2518

25. Bobrowski A, Nowak K, Zarebski J (2005) Application of a bismuth film electrode to the voltammetric determination of trace iron using a $\mathrm{Fe}(\mathrm{III})-\mathrm{TEA}-\mathrm{BrO}_{3}{ }^{-}$catalytic system. Anal Bioanal Chem 382:1691-1697

26. Segura R, Toral M, Arancibia V (2008) Determination of iron in water samples by adsorptive stripping voltammetry with a bismuth film electrode in the presence of 1-(2-piridylazo)-2-naphthol. Talanta 75:973-977

27. Lin M, Pan D, Hu X, Li F, Han H (2015) A tin-bismuth alloy electrode for the cathodic stripping voltammetric determination of iron in coastal waters. Anal Methods 7:5169-5174

28. Ugo P, Moretto LM, De Boni A, Scopece P, Mazzocchin GA (2002) Iron (II) andiron (III) determination by potentiometry and ionexchange voltammetry ationomer-coated electrodes. Anal Chim Acta 474:147-160

29. Gholivand MB, Geravandi B, Parvin MH (2011) Anodic stripping voltammetric determination of iron(II) at a carbon paste electrode modified with dithiodianiline (DTDA) and gold nanoparticles (GNP). Electroanalysis 23:1345-1351

30. Anguiano DI, Garcia MG, Ruiz C, Torres J, Alonso-Lemus I, Alvarez-Contreras L, Verde-Gomez Y, Bustos E (2012) Electrochemical detection of iron in a lixiviant solution of polluted soil using a modified glassy carbon electrode. Int J Electrochem 739408:6

31. Lin M, Han H, Pan D, Zhang H, Su Z (2015) Voltammetric determination of total dissolved iron in coastal waters using a glassy carbon electrode modified with reduced graphene oxide, Methylene Blue and gold nanoparticles. Microchim Acta 182: 805-813

32. Lin M, Pan D, Hu X, Han H, Li F (2015) Titanium carbide nanoparticles/ion-exchange polymer-based sensor for catalytic stripping determination of trace iron in coastal waters. Sensors Actuators B 219:164-170

33. Zarebski J (1977) Alkaline triethanoloamine-bromate solutions as supporting electrolytes for the determination of iron in trace amounts by differential pulse polarography. Chem Anal 22:10491051

34. Peng B, Shen YP, Gao ZT, Zhou M, Ma YJ, Zhao SG (2015) Determination of total iron in water and food by dispersive liquidliquid microextraction coupled with microvolume UV-vis spectrophotometry. Food Chem 176:188-293 